\title{
Use of a low nutrient culture medium for the identification of bacteria causing severe ocular infection
}

\author{
SIMON E. HORGAN, MELVILLE M. MATHESON*, LOUISE MCLOUGHLIN-BORLACE* and \\ JOHN K. DART
} Anterior Segment Service, Moorfields Eye Hospital, City Road, London and *Department of Microbiology,
Institute of Ophthalmology, London

\begin{abstract}
A low nutrient culture medium was used to identify the pathogens in four cases of persisting ocular infection. Bacto $\mathbf{R 2 A}$ agar was used in addition to conventional liquidand solid-phase media to culture pathogenic bacteria from one case of recurrent keratitis, one case of suture-related keratitis with endophthalmitis and two eyes (two patients) with post-operative endophthalmitis. In each case, a pathogen was identified solely with R2A agar after culture for 6 days. Species isolated were Pseudomonas aeruginosa (one), Propionibacterium acnes (two) and Staphylococcus aureus (one). Antibiotic therapy was tailored to conform to the sensitivity of the cultured organism in each case. The use of Bacto $\mathrm{R} 2 \mathrm{~A}$ low nutrient agar should be considered in culture negative eyes not showing clinical improvement, or for chronic cases where bacteria may have become adapted to more stringent ocular environments.
\end{abstract}

\section{Introduction}

The best management of ocular infection stipulates that knowledge of the infecting micro-organisms should determine appropriate antimicrobial treatment [1-4]. Conventionally, media rich in proteinaceous substrates (typically blood, meat and yeast extracts) are used to culture material from infected eyes and it is assumed that pathogens will proliferate under optimal conditions. However, some bacteria exist in clinical environments very different from these highly nourished laboratory conditions [5] and may even reside within the eye as biofilms $[6,7]$. These micro-environments may sustain bacteria adapted to more stringent conditions that will not necessarily be cultured by standard methods. Consequently, there is a need for a culture medium which allows recovery of slow-growing pathogenic bacteria.

Bacto R2A agar (Difco Laboratories, Detroit, MI, USA) is a low nutrient medium whose development arose from the requirements of public health laboratories and water companies to satisfy standards of water purification [8]. Conventional nutritionally rich media such as Plate Count Agar and Tryptone Yeast

Received 29 Sept. 1998; revised version received 30 Nov. 1998; accepted 2 Dec. 1998.

Corresponding author: Mr S. E. Horgan.
Extract agar failed to recover all bacteria from treated potable water, and Bacto R2A agar was introduced to meet these requirements [8]. Low concentrations of nutrients such as starch $(0.5 \mathrm{~g} / \mathrm{L})$ and dextrose $(0.5 \mathrm{~g} / \mathrm{L})$ favour slow-growing bacteria, whilst the presence of sodium pyruvate $(0.3 \mathrm{~g} / \mathrm{L})$ increases recovery of stressed organisms.

We believe that this paper provides the first report on the use of a low nutrient agar in a clinical setting to identify pathogens responsible for persisting ocular infection.

Case histories, methods and results

Case 1

A 47-year-old man with a history of severe ocular surface disease and previous corneal grafting presented with presumed stromal keratitis at the graft-host interface in one eye. The lesion was inaccessible for scraping, but keratectomy specimens were sent for standard culture. Both Gram's and Grocott-Gomori stains of keratectomy specimens showed diphtheroidtype organisms, but positive identification of Propionibacterium acnes was achieved only with R2A agar (no culture was obtained with thioglycolate broth, brain-heart infusion broth, cooked meat broth and Sabouraud's dextrose agar). Positive identification of a 
pathogen confirmed the need for antibiotic as well as corticosteroid therapy. The patient received a 3-day course of azithromycin and his long-term treatment was modified following this positive culture result.

\section{Case 2}

This was a case of endophthalmitis referred from another hospital 2 days after cataract surgery with lens implantation. Acuity had fallen to hand motions in the presence of $1 \mathrm{~mm}$ hypopyon and the patient had already been treated with oral and topical ciprofloxacin. Aqueous and vitreous taps were undertaken at presentation and whilst no bacteria were identified with conventional media, Pseudomonas aeruginosa was cultured with R2A agar after incubation for 6 days at $30^{\circ} \mathrm{C}$. Gram's stain revealed gram-negative bacilli consistent with $P$ s. aeruginosa. Clinical improvement followed repeated use of intracameral amikacin $0.4 \mathrm{mg}$ and intensive ofloxacin, with vision improving to $6 / 18$.

\section{Case 3}

This 75-year-old patient underwent cataract surgery and lens implantation, but developed chronic endophthalmitis. Appropriate initial investigation was inconclusive and empirical antibiotic treatment was unsuccessful. The patient underwent vitrectomy, capsulectomy and received intravitreal vancomycin $2 \mathrm{mg}$ with some improvement, but as there was persisting infiltrate at the capsule margin, she needed a complete capsulectomy. P. acnes was cultured from vitreous and aqueous specimens on R2A agar, but this organism was not isolated with conventional media. Examination of capsular fragments showed pleomorphic gram-positive organisms on Gram's and Grocott-Gomori stains that were morphologically indistinguishable from propionibacteria. Confirmation of $P$. acnes-associated chronic endophthalmitis indicated that long-term antibiotic therapy was advisable following surgical excision of the infected intra-ocular material.

\section{Case 4}

A 75-year-old patient had undergone combined corneal graft and cataract surgery. Five months later she developed an area of keratitis surrounding a loosened nylon suture. The eye also exhibited a low grade uveitis with vitreous opacities and the possibility of endophthalmitis was considered. Aqueous and vitreous taps and a stitch abscess scrape were performed, together with culture of the loosened suture material. Staphylococcus aureus and Haemophilus influenzae were grown from the corneal scrape with conventional and R2A media, but only $S$. aureus was cultured from aqueous with R2A (not isolated on conventional media). Insufficient vitreous was obtained for culture. Gram-positive cocci, morphologically indistinguishable from staphylococci were also identified on microscopy.
Although the keratitis was polymicrobial, the confirmation of $S$. aureus from intra-ocular material suggested that $H$. influenzae may have been a contaminant. Intracameral vancomycin and amikacin together with oral ciprofloxacin and clarithromycin were used and the infection resolved, with acuity improving to $6 / 24$.

\section{Microbiological techniques}

In cases of keratitis, specimens were obtained following instillation of two drops of unpreserved topical anaesthetic. The infected area was scraped with $21 \mathrm{G}$ needles and the debris was inoculated directly from the needle tip onto the solid media, R2A medium and Sabouraud's dextrose agar. For liquid-phase media (cooked meat broth, thioglycolate broth and brain-heart infusion broth) the sample on the needle tip was transferred on to swabs moistened in the media and then broken off into the culture bottles without contaminating the shafts. Good aseptic technique was observed throughout. Additional scrape material was placed on to a microscope slide and air-dried for microscopy.

For cases of endophthalmitis, specimens were obtained in the operating theatre. The conjunctival sac was disinfected with two drops of aqueous povidone-iodine and $0.1 \mathrm{ml}$ of fluid was removed through an anterior chamber paracentesis with an insulin syringe. A 0.2$\mathrm{ml}$ sample of vitreous was obtained by pars plana needle puncture or with a vitreous suction cutter. The samples were transferred to culture media as for keratitis specimens.

Culture media were incubated at $37^{\circ} \mathrm{C}$ in air with $\mathrm{CO}_{2}$ $5 \%$ except for R2A agar, which was incubated at $30^{\circ} \mathrm{C}$ and Sabouraud's dextrose agar at $25^{\circ} \mathrm{C}$. All liquid media were subcultured twice over a period of $72 \mathrm{~h}$. Positive growth from one medium was considered to be significant where there was consistency with the findings from microscopy. All cultured organisms were tested for susceptibility to aminoglycosides, fluoroquinolones, penicillin and cephalosporins.

\section{Discussion}

Microbial keratitis and endophthalmitis are blinding conditions. Early diagnosis and treatment are important for successful treatment, although age, pathogenicity of the organism and presenting visual acuity are useful indicators of outcome [2,9,10-12]. Despite the efficacy and good tolerance of broad-spectrum antimicrobial agents, laboratory investigation of the pathogens should be regarded as integral to a rational approach to management $[2,3,13,14]$.

Even for tertiary referral centres with dedicated microbiological facilities, the culture positivity rate 
for endophthalmitis is only $62-69 \%[4,9]$ and for keratitis $40-74 \%[1,12,15,16]$. These centres tend to manage more severe or recalcitrant infections and also have a duty to record the nosological epidemiology of local disease [14]. In this context the use of a low nutrient medium may provide an increased rate of positive cultures which, in turn, will favour clinical outcomes.

Culture-negative cases of keratitis may represent either sterile infiltrates or infected eyes in which pathogens are not cultured by conventional methods. The four cases described here illustrate the difficulty sometimes experienced in obtaining positive culture results, particularly when eyes have already been treated with antibiotics, and in instances where distinction between inflammation and infection is clinically difficult (e.g., cases 1 and 3 ).

It is unlikely that the organisms obtained on R2A culture were contaminants, as they were consistent with morphology seen on Gram's staining of the same material. The four cases described represent part of a series of 20 separate ocular isolates in which pathogens were cultured consistently on R2A agar as well as being grown on conventional media. To date there have been no instances of culture negativity on R2A agar and positive culture on conventional media. Consequently, it appears that R2A agar increases the sensitivity and specificity of microbiological culture in selected cases. At present such cases comprise those with chronic or relapsing infection, or eyes presenting acutely in which appropriate initial investigation has proved fruitless.

Some evidence exists to show that pathogenic organisms become adapted to more stringent ocular environments, making conventional culture difficult. Eyes with endophthalmitis may contain bacteria encapsulated within biofilms $[6,7]$ and vitreous could be regarded as a relatively low nutrient substance. Keratitis may exist deep within devitalised corneas or in eyes being treated with prolonged topical antibiotics and corticosteroid. Consequently, there is a role for low nutrient culture media in the microbiological isolation of pathogens, and this should not be seen as an alternative to conventional media, but as complimentary to them. The present study used R2A agar plates, which require no special preparation and only need incubating at $30^{\circ} \mathrm{C}$. The benefit of microbiological work-up in cases of keratitis is debatable at present, given the potency of currently available widespectrum antibiotics [3]. However, in severe or relapsing cases of infection that may be unresponsive to conventional therapy, as in three of the cases described above, cultures are an integral part of successful management. An increased culture positivity is achievable with low nutrient agar in cases of keratitis and endophthalmitis.

None of the authors has propietary or financial interests in the materials or techniques described above.

\section{References}

1. McLeod SD, Kolahdouz-Isfahani A, Rostamian K, Flowers CW, Lee PP, McDonnell PJ. The role of smears, cultures, and antibiotic sensitivity testing in the management of suspected infectious keratitis. Ophthalmology 1996; 103: 23-28.

2. Allan BDS, Dart JKG. Strategies for the management of microbial keratitis. $\mathrm{Br} J$ Ophthalmol 1995; 79: 777-786.

3. McDonnell PJ. Empirical or culture-guided therapy for microbial keratitis? A plea for data. Arch Ophthalmol 1996; 114: $84-87$.

4. The Endophthalmitis Vitrectomy Study Group. Microbiologic factors and visual outcome in the Endophthalmitis Vitrectomy Study. Am J Ophthalmol 1996; 122: 830-846.

5. Laws EA, Jones D, Karl DM. Method for assessing heterogeneity in turnover rates within microbial communities. Appl Environ Microbiol 1986; 52: 866-874.

6. Elder MJ, Stapleton F, Evans E, Dart JKG. Biofilm-related infections in ophthalmology. Eye 1995; 9: 102-109.

7. Morlet N, McLaughlin-Borlace L, Dart J. Bacterial biofilm on explanted intraocular lenses (IOLs). Invest Ophthalmol Vis Sci 1996; 37: S758.

8. Reasoner DJ, Geldreich EE. A new medium for the enumeration and subculture of bacteria from potable water. Appl Environ Microbiol 1985; 49: 1-7.

9. Bohigian GM, Olk RJ. Factors associated with a poor visual result in endophthalmitis. Am $J$ Ophthalmol 1986; 101: $332-334$.

10. Salvanet-Bouccara A, Forestier F, Coscas G, Adenis JP, Denis F. Endophtalmies bacteriennes. Resultats ophtalmologiques d'une enquete prospective multicentrique nationale. [Bacterial endophthalmitis. Ophthalmological results of a national multicentre prospective survey.] $J$ Fr Ophtalmol 1992; 15: 669-678.

11. Han DP, Wisniewski SR, Wilson LA, Barza M et al. Spectrum and susceptibilities of microbiologic isolates in the Endophthalmitis Vitrectomy Study. Am J Ophthalmol 1996; 122: 1-17.

12. The Ofloxacin Study Group. Ofloxacin monotherapy for the primary treatment of microbial keratitis: a double-masked, randomized, controlled trial with conventional dual therapy. Ophthalmology 1997; 104: 1902-1909.

13. Armstrong $M$. The laboratory investigation of infective keratitis. Br J Biomed Sci 1994; 51: 65-72.

14. Allan BD, Morlet N, Dart JK. Microbiologic investigation of suspected microbial keratitis. Ophthalmology 1996; 103: 11651166.

15. Liesegang TJ, Forster RK. Spectrum of microbial keratitis in South Florida. Am J Ophthalmol 1980; 90: 38-47.

16. Galentine PG, Cohen EJ, Laibson PR, Adams CP, Michand R, Arentsen JJ. Corneal ulcers associated with contact lens wear. Arch Ophthalmol 1984; 102: 891-894. 